\title{
Preparing Teaching Materials for Engineering Disciplines with Markdown
}

\author{
Tuoxin Jiang \\ Faculty of Foreign Languages and Cultures \\ Kunming University of Science and Technology \\ Yunnan, PR China \\ kmjtx@126.com
}

\author{
Xueying Liu \\ Faculty of Electric Power Engineering \\ Kunming University of Science and Technology \\ Yunnan, PR China \\ 573409874@qq.com
}

\author{
Lan Tang \\ Faculty of Electric Power Engineering \\ Kunming University of Science and Technology \\ Yunnan, PR China \\ tanglan@kmust.edu.cn
}

\begin{abstract}
In the digital age, teachers in engineering disciplines must pay more attention to the preparation of teaching materials than before. However, their work efficiency is limited by words processing because of the complexity of teaching materials that consist of text, equations, figures, etc. A method based on the plain text is presented in this paper. The common syntax of Markdown is introduced, two practical MD editors and a useful tool, Pandoc, are recommended.
\end{abstract}

Keywords-teaching materials, markup language, Markdown, Pandoc, efficiency

\section{INTRODUCTION}

With the development of network technology and the changing of teaching theory, engineering courses in colleges are beyond lectures, homework and design projects. Students could read teaching materials and watch teaching video to further study via network. Therefore, teachers not only need making preparation for lectures but also supplying lots of learning materials for students, which is one of the indispensable responsibilities of college teachers. Nowadays, "Flipped classroom" becomes more and more popular, which highlights the importance of preparing electronic teaching materials [1]. In China, most of the teachers choose a "WYSWYG" tool as text editor, such as MS word or WPS. When many mathematical symbols and formulas need to be input, it becomes a time-consuming job to type in teaching materials even with the help of a visual plugin. And files with the proprietary format are difficult to be released on the network. Sometimes, students could download files to local computer, but fail to open them because of the differences between reader and original editor. Although a few teachers may utilize LaTeX typing system to make e-documents, which is a good way to solve the problem above mentioned, it has not been widely used in China.

In this paper, Markdown-based text processing was introduced. Both mathematical symbols and figures could be inserted into the plain text conveniently. The MD files could be converted to many different formats, and more suitable to be posted on the net. Besides these advantages, anyone could learn Markdown in ten minutes and master it during the course of practice.

\section{WHAT MARKDOWN IS}

Markdown is a lightweight markup language with plain text formatting syntax designed so that it can be converted to HTML and many other formats [2,3]. Markdown usually has two means. One is the syntax; the other is the converting tool. The key design goal of Markdown is readability. A Markdownformatted document should be publishable as-is, as plain text, without looking like it's been marked up with tags or formatting instructions. Therefore, Markdown was first popular in computer society and often used to format readme files, for writing messages in online discussion forums. For example, Markdown is supported by famous WordPress and Github.

There is no clearly defined Markdown standard till now. Many implementations extend Markdown by adding features (such as tables, footnotes, definition lists, mathematics, and Markdown inside HTML blocks) not available in original version [3]. Pandoc's enhanced version of Markdown is the typical one. Because of these extensions, markdown could be used as a good tool to edit teaching materials.

In this section, a little syntax was introduced at the beginning. As the matter of fact, only by using title mark and list mark could we write general articles without thinking of the format any more.

\section{A. Basic symbols}

There are three kinds of symbols in Markdown. They are ordinary markers, blanks and special segments markers. 


\section{1) Ordinary markers}

There are three ordinary markers, asterisks, pluses and hyphens (*,+,-). These three markers are interchangeable. Of course, keeping consistency in a file is a good habit.

\section{2) Blanks}

Two blanks appeared at the end of a line means return. One or more blank lines are used to separate one paragraph from another.

\section{3) Special segments markers}

In a regular paragraph, you can create code span by wrapping text in backtick quotes (').To specify an entire block of pre-formatted code; indent every line of the block by 4 spaces or 1 tab.

\section{B. Headers}

The structure of a paper can be presented by hierarchical headers. Headers in different hierarchies are distinguished with different fonts or word size. Markdown offers two styles of headers: Setext and atx. Setext-style headers for $<$ h $1>$ and $<$ h $2>$ are created by "underlining" with equal signs $(=)$ and hyphens (-), respectively. To create an atx-style header, you put 1-6 hash marks (\#) at the beginning of the line - the number of hashes equals the resulting HTML header level.

\section{Lists}

Unordered lists use any one of basic symbols as list markers. Ordered lists use regular numbers, followed by periods, as list markers. Nested lists are also supported.

\section{MARKDOWN EDITORS}

The name of document marked with Markdown is usually ended with .MD. It is still a plain text file. Therefore, almost all the text editors could be used to make MD document. There are specially designed editors that preview the files with styles, which could be grouped into three categories: general purpose editors with markdown plugins, online MD editors and special desktop application.

Many general purpose text and code editors, such as Vim, Sublime Text, Eclipse, etc., have syntax highlighting plugins for Markdown built into them or available as optional download, which are too complicated except for users with background of programming.

Both online and stand-alone editors may feature a side-byside preview window or try to render the code directly in a WYSIWYG-like fashion. Each editor is with different design goal and has its own characters. In view of its cross-platform, Chinese compatibility and usability, Cmd Markdown and Haroopad are recommended. To edit rich text as teaching materials, both of them are available.

Cmd Markdown was developed by zybuluo.com, and could be used as an online editor or an offline client. It has many features that make it easier to edit technology document. As the author said, "we supply professional tool for taking notes of thought and sharing knowledge.”
Haroopad is an open source project hosted on Github. Its purpose is providing a professional tool for document making on internet. It is a cross-platform desktop application. Compared with Cmd markdown, there are many basic syntax items in the Haroopad's insert menu. It is really helpful to the newbie.

\section{TRICKS FOR MAKING OF TEACHING MATERIALS}

\section{A. Features of teaching materials for engineering disciplines}

Compared with general network-article, there are more salient features in teaching materials for engineering disciplines besides clear structure, good format and cross-platform compatibility. These features are: (1) a mass of formulas that are difficult to be input in files; (2) various objects embedded in files as tables, figures and curves; (3) special segments containing codes or citations in files. Teaching materials are typical of rich text.

\section{B. Inserting mathematical formulas}

Processing mathematical formulas is the bottleneck of editors of "WYSWYG". A user has to move his hand between keyboard and mouse constantly while inputting equations, which leads to low efficiency. Words and formulas appeared in the same line make line spacing changed, which destroys the format of files. The defect of editors is one of the reasons why network teaching system fails to attract teacher from engineering disciplines [4].

Cmd Markdown recommended in this paper has been integrated with MathJax, which displays mathematical notation in web browsers, using MathML, LaTeX and ASCII Math ML markup. Thus, an author could edit mathematical formulas keeping his hand on the keyboard without caring about the format of the file.

Generally speaking, there are two kinds of formulas. One is inline formula that is written in the paragraph such as $\int_{-\infty}^{\infty} \frac{1}{\sqrt{2 \pi}} e^{-\frac{(x-\mu)^{2}}{2 \delta^{2}}} d x=1$. independent with counting number as eq.1.

$$
\int_{-\infty}^{\infty} \frac{1}{\sqrt{2 \pi}} e^{-\frac{(x-\mu)^{2}}{2 \delta^{2}}} d x=1
$$

In MD file, both of them are a string of characters in math mode: \int $\wedge \backslash$ infty_\{-linfty $\} \backslash$ frac $\{1\}\{\backslash$ sqrt $\{2 \backslash$ pi $\}\} \mathrm{e}^{\wedge}\{-\backslash$ frac $\{(\mathrm{x}-$ $\left.(\mathrm{mu}) \wedge 2\}\left\{2 \backslash \operatorname{delta}^{\wedge} \wedge 2\right\}\right\} \mathrm{dx}=1$.

So-called math mode is a segment of text between starting characters and ending characters. About the definition of the starting and ending characters, different MD editors have their own choices. For example, a ' $\$$ ' is used as the beginning and the ending of inline math segment by Cmd Markdown, while explicit formula environment is built by two “\$\$”. “\$\$” defined by Haroopad is used to implement the same function. So we have to read the manual of the editor to get details. Of course, much patience is needed to be familiar with any one set of the syntax for mathematical notations markup. Among three mathematical markup languages above mentioned, AMS Math 
[3] MarkDown, https://en.wikipedia.org/wiki/Markdown.

[4] Misfeldt M, Sanne A. Formula Editors and Handwriting in Mathematical E-Learning, Teaching Mathematic Online Emergent and Technologies and Methodologies, pp.350-364, 2012.
[5] George Gratzer, More Math Into LaTeX(5th edition), Springer, 2016

[6] Pandoc, http://www.Pandoc.org 